\title{
Sexualidad femenina y versiones del final de análisis en la práctica clínica con niños ${ }^{*}$
}

\author{
Female sexuality and versions of the end of analysis in clinical \\ practice with children

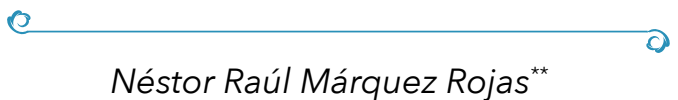 \\ Néstor Raúl Márquez Rojas

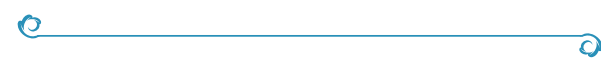

Recibido: $15.10 .2018 \bullet$ Arbitrado: 08.11.2018•

Aprobado: 05.12.2018

Articulo derivado de la investigación para otra el título de Doctor en Ciencias Sociales.

* Psicólogo, Magister en Psicoanálisis, Estudiante de Doctorado en Ciencias Sociales; Profesor de tiempo completo, Facultad de Ciencias Sociales, Institución Universitaria de Envigado, ORCID: nrmarquez@correo.iue.edu.co

\section{Resumen}

El abordaje de la familia desde la perspectiva del psicoanálisis no se orienta a una intervención de carácter grupal, sino que tiene como centro de atención las demandas de cada uno de los integrantes del colectivo familiar. En un proceso de análisis no se escucha al grupo familiar, sino las fantasías del padre, la madre y el niño. Este último en particular merece una atención especial en el dispositivo analítico, ya que su demanda en la experiencia clínica, obedece al axioma lacaniano, según el cual, "el síntoma del niño está en posición de responder a lo que hay de sintomático en la estructura familiar". En el inicio de dicha experiencia clínica con el niño, por lo general, la demanda siempre procede de un Otro de la familia, en particular la madre. Pero a diferencia de Klein, o de Winnicott, Lacan fue muy contundente al señalar que la experiencia de un análisis con niños no se debía de reducir por la vía del maternaje. Su propuesta en este sentido es revolucionaria, ya que propone una vía de intervención con el niño a través del vínculo de la "sexualidad femenina y sus relaciones con el objeto niño". En esta práctica 
clínica no se trata entonces de reducir el análisis a un proceso meramente pedagógico; de lo que se trata es de reconocer que él niño, en tanto sujeto, también está implicado con su síntoma al Otro. El proceso de análisis se orienta no sólo para abordar el síntoma que él representa para la pareja de los padres, sino del síntoma que él inventa para dar cuenta de una experiencia singular, esto es, una experiencia de goce singular con los objetos más cercanos. Por ello, el síntoma del niño se estructura, así como una metáfora familiar.

Palabras clave: familia, final de análisis, fantasma, sujeto, síntoma, psicoanálisis.

\section{Abstract}

The approach of the family from the psychoanalysis perspective is not aimed towards a group nature intervention, instead, it focuses on the demands of each of the members of the family group. In an analysis process, the family group is not heard, but the fantasies of the father, mother and child are. The latter in particular deserves special attention in the analytical mechanism, since its demand in clinical experience, obeys the Lacanian axiom, according to which, "the child's symptom is in a position to respond to what is symptomatic in the family structure". At the beginning of this clinical experience with the child, usually the demand always comes from an Other of the family, in particular the mother. But unlike Klein, or Winnicott, Lacan was very firm in pointing out that the experience of an analysis with children should not be reduced by the maternal way. In this sense, his proposal is revolutionary, since he proposes a way of intervention with the child through the bond of "female sexuality and its relations with the child object". In this clinical practice, the intention is not to reduce the analysis to a merely pedagogical process; it is about recognizing that the child, as a subject, is also implicated with his symptom to the Other. The analysis process is oriented not only to address the symptom he represents to the parents, but also the symptom he invents to account for a singular experience, that is, an experience of singular enjoyment with the closest objects. Therefore, the child's symptom is structured as a family metaphor.

Keywords: family, final analysis, ghost, subject, symptom, psychoanalysis.

\section{Introducción}

El presente escrito está orientado en el campo de la clínica y tiene como propósito fundamental anudar los ejes específicos sobre los cuales gira el psicoanálisis en general, y la practica con niños en particular, estos son: sexualidad femenina, práctica clínica con niños y las versiones del final de análisis en el 
niño. Muy tempranamente Freud a través de su trabajo "Tres ensayos para una teoría sexual" (1907), articula los ejes que orientan la práctica clínica; en general y al mismo tiempo, es lo decisivo en el psicoanálisis con niños. Veamos entonces como se desarrolla a lo largo de cinco tesis de trabajo esta relación de práctica clínica con niños, sexualidad femenina y sus nexos con las versiones del final de análisis.

En su trabajo "La sexualidad femenina: un recorrido a través de la teoría fálica", Freud nos enseña acerca de las vicisitudes que tiene que sortear el sujeto femenino en relación al núcleo edipico y la castración. La sexualidad humana según el psicoanálisis no se rige por el instinto sino por el fantasma que contiene una estructura de carácter perverso. Así la sexualidad en el niño, en términos de Trieb, desde "Tres ensayos..." está articulada con la perversión y, después, con el fantasma.

El estatuto de la sexualidad femenina tal como lo destaco Freud, deja ver muchos puntos enigmáticos, y esto porque el Edipo en la niña se concibe de manera contraria al Edipo en el niño. Freud a través del estudio de casos de histeria femenina, llega a la deducción de que, "algo de lo no concluido es especifico de la sexualidad femenina". En su reflexión "Sobre la sexualidad femenina", Freud plantea una simetría entre hombres y mujeres respecto de la premisa fálica. Propone para la mujer tres orientaciones del desarrollo: una la inhibición sexual o la neurosis, la otra, la alteración del carácter en el sentido de la masculinidad, y la tercera la feminidad normal; vía de la ecuación simbólica pene $=$ hijo.

A partir de estas tres orientaciones que propone Freud, se rastrea el nexo: neurosis infantil y sexualidad femenina. Para el desarrollo de este punto se partió de la elaboración axiomática de Freud, en el sentido de que, "toda neurosis del adulto se construye sobre las reliquias de una neurosis infantil" Silvestre (1987). Un caso prínceps de Freud, el estudio del "Hombre de los lobos" (1918), ejemplifica ésta elaboración. Así mismo, a través del análisis de un caso de histeria femenina, "Dora" (1907); y un caso de obsesión masculina, "Hombre de las ratas" (1910), se puede palpar el nexo neurosis infantil y sexualidad femenina. Destacando particularmente en uno y otro caso una versión posible del final de análisis, así se tendría que: "tanto para los hombres como para las mujeres el final de análisis se presenta como un callejón sin salida, y esto porque el encuentro con la feminidad puede constituir un encuentro con lo real. Encuentro con lo real, en tanto lo que se pone en juego para el sujeto es "la realidad inconsciente del significante falo" (Miller, 1988).

Estas reflexiones sobre el Edipo en la mujer tratan fundamentalmente de la falta femenina, de la división que produce el objeto-niño en tanto dicha falta 
se trata de resarcir ya bien por la vía de la maternidad, o por vía alterna que implica un goce suplementario ligado a la mujer y no a la madre. Esta cuestión es preliminar a todo tratamiento posible con los niños: "en la clínica se debe tener en cuenta que el niño tiene una relación especial con la madre, y que la madre es ante todo una mujer". La anterior elaboración clínica surge a propósito del estudio que realiza Jacques Lacan en el seminario "La relación de objeto" (1957), en donde pone en el centro de la problemática infantil la relación madre-niño. Lacan hace hincapié en el hecho de que, "todo síntoma infantil tiene que ver con la subjetividad de la madre, en tanto que la madre es una mujer". Todas las directrices de la clínica con niños se orientan a partir de la posición que ocupa el niño en relación con la posición femenina de la madre. Y, ello porque la madre, como mujer, tiene una relación especial con la falta, de un objeto primordial, que desde el trabajo sobre la "sexualidad femenina" Freud (1921), denominó como falo. Con este objeto, la mujer tiene una relación fundamental y es con este objeto que el niño va a introducirse en la subjetividad de la madre.

La anterior reflexión lleva a plantear los nexos entre sexualidad femenina, en términos de la metáfora del Nombre-del-Padre y las versiones o modalidades del final de análisis con niños. Para ello inicialmente se rastrean a través de S. Freud y J. Lacan la noción de final de análisis y sus implicaciones en la clínica con niños. Luego se realiza una reflexión desde el punto de vista de la teoría fálica y el -objeto a-sobre las versiones del final de análisis. Y, finalmente se desarrolla la conexión: Sexualidad femenina, práctica clínica con niños y las versiones del final de análisis. Sobre estos dos ejes fundamentales, sexualidad femenina en términos del deseo de la madre (DM); y la metáfora del niño, objeto sustituto, Ersatz (sustituto) del deseo materno, se pueden orientar las distintas versiones del final de análisis en la clínica con niños. Y, es acá que cobra valor la pregunta que orienta el presente ensayo, esto es: ¿Qué incidencias en el final de análisis con niños tiene la sexualidad femenina?

Jacques Alain Miller (1988), insiste en que, "cada análisis de niños implica verificar como el sujeto - niño (Sn) se articulan en relación al sujeto - femenino ( $S f$ ) con su falta fálica, y como se inscribe el niño en esa relación". Esto implica exponer las consecuencias clínicas de la sexualidad femenina para cada sujeto, en la medida en que cada uno es hijo de una madre. Por ello el tema más importante para trabajar en la clínica con niños, es el tema de la sexualidad femenina.

A partir de las elaboraciones sobre la metáfora fálica en Freud y la conceptualización del objeto $\boldsymbol{a}$ en Lacan, se pueden orientar dos versiones del final 
de análisis con niños: una en donde el niño tenga una versión del falo- instante de la sexuación del infantil sujeto - ; otra en la cual el niño tenga una versión del objeto $\boldsymbol{a}$, construcción de un fantasma que garantice que su cuerpo no será condensador del goce materno. Y, por último, "el final de análisis con niños implica un tratamiento del síntoma y su implicación en el fantasma". Acá hay una doble doctrina sobre la práctica clínica con niños, según si el análisis se conciba como medio para alcanzar una mediación simbólica incluso subjetiva del niño en el fantasma, o como operación de desciframiento del goce del síntoma.

\section{Metodología}

Para la realización del presente artículo, se utilizó el enfoque cualitativo, nivel descriptivo y método bibliográfico, el cual tiene por propósito, según Alfonso (1995), indagar, recolectar, organizar, analizar e interpretar información o datos en torno a una determinada temática. Este tipo de trabajo se articula dentro de las técnicas de investigación documental, las cuales se refieren por lo general a las fuentes de información utilizadas en la investigación y se le denominan genéricamente como "Unidades Conservatorias de Información" y se trata de documentos, bibliografías, publicaciones, Estados del Arte, Estados del Conocimiento, y textos de autores de primera mano, cuya función es la de almacenar o contener información. Estas técnicas de investigación documental se aplican a algunos tipos de documentos -generalmente a textos: libros, artículos en revistas y reportes de entrevistas, entre otros- (Rojas Crotte, 2011:281).

Para la elaboración del presente ensayo se seleccionaron los trabajos originales de aquellos autores del campo del psicoanálisis contemporáneo Miller, Laurent, Solano, y Silvestre; de otro lado, se revisaron autores clásicos como Freud, Winnicott y Lacan. Esta revisión se llevó a cabo a través de la disciplina del comentario de texto. La lectura intratextual es un primer tiempo de lectura que aspira a investigar un texto, para intentar establecer, sólo desde el texto mismo, lo que éste dice. En tanto que, la lectura intertextual, comprende el segundo tiempo de la lectura, en el cual se pretende cotejar y someter a discusión unidades de análisis (párrafos, conceptos, enunciados, etc.) de dos o más textos, de uno o varios autores (Pérez, 1998:239).

Como criterios de selección de los respectivos trabajos, se tuvo en consideración el "Criterio de pertinencia", esto es, que las fuentes consultadas deben ser acordes con el objeto de investigación y con sus respectivos objetivos, en tanto que estos aportan conocimientos, enfoques, teorías, conceptos y 
experiencias significativas para fundamentar el tema de estudio. De otro lado, se tomó en consideración el "Criterio de actualidad", el que, a su vez, implica que las fuentes consultadas deben ser lo suficientemente actuales como para asegurar que reflejen los últimos desarrollos de la materia, o disciplina objeto de tratamiento. Por último, se tuvieron en consideración los aspectos éticos relacionados con los derechos de autor y las respectivas normas de citación exigidas por la APA (2006).

\section{Desarrollo del tema}

\section{Acerca del final de análisis con niños y sus nexos con la sexuali-} dad femenina: la teoría fálica y la teoría del objeto a”.

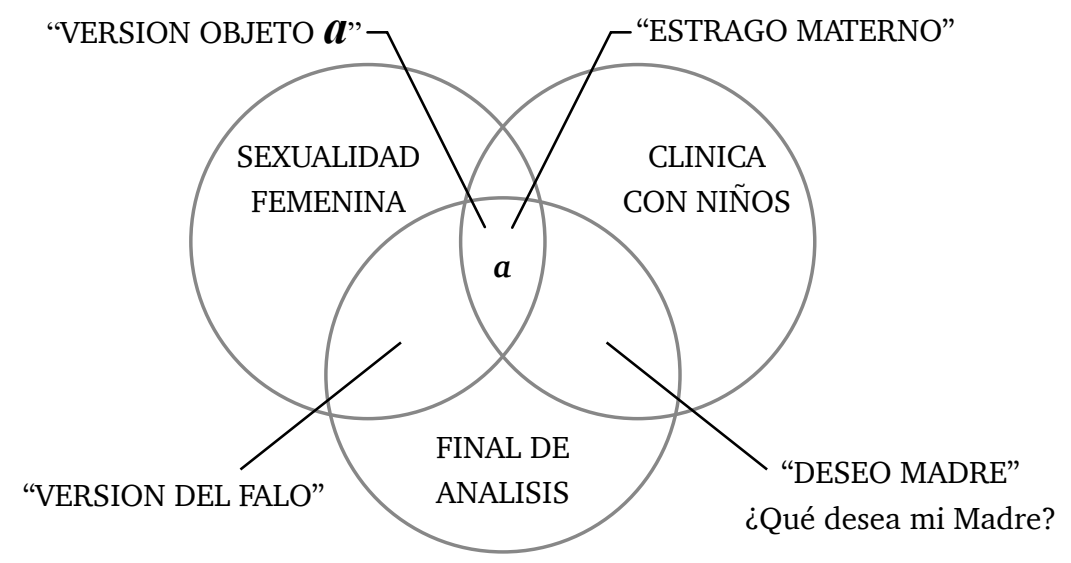

Figura N 1: Práctica clínica con niños

La clínica psicoanalítica según Lacan tiene su fundamentación en dos teorías: la teoría fálica elaborada por S. Freud, y la teoría del objeto $\boldsymbol{a}$; la cual Lacan considera su única invención en psicoanálisis. Por ello la pregunta que orienta el presente texto, se podría articular en los siguientes términos: ¿Qué incidencias en el final de análisis con niños tiene la sexualidad femenina? La respuesta a dicha pregunta se va a encontrar en las dos teorías antes anunciadas, las cuales según J.A. Miller en "Recorrido de Lacan" (1998), "La lógica de una cura" (2011), y en "Dilucidación de Lacan" (1988), se pueden descomponer a su vez en distintas tesis de trabajo, las cuales dejan entrever el fin de análisis en la clínica con niños; veamos cuales son y qué lugar ocupan en la pregunta que el presente ensayo busca dilucidar. 


\section{Primera tesis: "la clínica freudiana es ante todo una clínica del falo"}

La clínica freudiana es ante todo una clínica del falo: para ello Lacan retoma de Freud la tesis del complejo de Edipo y complejo de castración. La clínica freudiana, tal como Lacan la demuestra es, respecto a este punto retroactiva. Un ejemplo muy particular es la lectura que hace Lacan del sueño de la "Bella carnicera" (1957); allí va a releer ese sueño a partir de la función de la castración en la mujer. Este punto es capital en la clínica freudiana, y ello por dos elementos fundamentales: en primer lugar, la concepción de la sexualidad femenina, especialmente la función de la castración materna. En segundo lugar, la noción de deseo en tanto insatisfecho, en tanto correlativo a una falta.

El concepto de deseo es fundamental para articularlo en la clínica del falo, porque a partir de allí se orientarán las etapas propias de una cura. Eso introduce allí la función de la falta fundamental, de manera que en su seminario "La relación de objeto" (1957), lo primero que hace Lacan es presentar la falta de objeto; su primer gesto teórico es hacer girar esa cuestión hacia la teoría de la falta de objeto, que estará siempre perdido, como dice Freud en su elaboración en los "Tres ensayos de teoría sexual" (1905): el objeto fundamental es la madre y se pierde. Para Lacan, la noción de "relación de objeto" es imposible entenderla, incluso ejercerla, sino se introduce en falo, como uno de sus elementos orientadores, como el pivote de la clínica. "No mediador, porque eso sería dar un paso que todavía no hemos dado juntos, sino tercero" (Lacan, 1994: 30).

Considerando a la castración como nombre de la falta fundamental, la clínica freudiana opera alrededor de este concepto. Es por esto que, Lacan hace girar toda la teoría del objeto a partir de $(-\varphi)$, escritura del complejo de castración. "Nunca en nuestro ejercicio concreto de la teoría analítica, podemos prescindir de una noción de la falta de objeto con carácter central. No es negativa, sino el propio motor de la relación del sujeto con el mundo". Y luego agrega: "desde sus inicios, el análisis, el análisis de la neurosis empieza con la noción de castración" (Lacan, 1994: 39).

Segunda tesis: "la sexualidad femenina guarda sus nexos con la teoría fálica"

\section{Primer punto}

Para Lacan, el personaje central sobre el cual gira la teoría de la relación de objeto es la madre. El seminario de "La relación objeto" (1957), expone las consecuencias clínicas de la sexualidad femenina para todo sujeto, en la medida en que cada uno es hijo de una madre. "Por ello a partir de Lacan se 
puede sustentar que lo más importante para trabajar el psicoanálisis con niños, es el tema de la sexualidad femenina" (Miller, 1988:437).

Para Freud en el mundo de los objetos hay uno con una función paradójicamente decisiva, el falo. Este objeto se define según Lacan como imaginario, de ningún modo puede confundirse con el pene en su realidad, es propiamente su forma, su imagen erecta. Este falo tiene un papel tan decisivo, que tanto su nostalgia como su presencia, o su instancia en lo imaginario, "resultan al parecer más importantes todavía para los miembros de la humanidad a quienes les falta su correlato real, o sea a las mujeres" (Lacan, 1994:72). De otra parte, para Freud entre las faltas de objeto esenciales de la mujer está incluido el falo, y esto está íntimamente vinculado a su relación con el niño. Por una simple razón, si la mujer encuentra en el niño una satisfacción, es precisamente en la medida en que halla en él algo que calma, algo que satura, más o menos bien su necesidad de falo.

Lacan articula esta relación de la siguiente manera: "Lejos de ser armónica, la relación de la madre con el niño es doble, con, por una parte, una necesidad de cierta saturación imaginaria y, por otra parte, lo que pueden ser en efecto las relaciones reales y eficientes con el niño, en un nivel primordial, instintivo, que en definitiva resulta ser mítico". Por ello para la madre, siempre hay algo que permanece irreductible en esta relación, si se sigue a Freud, se puede decir que el niño como real simboliza la imagen. "Más precisamente - el niño como real ocupa para la madre la función simbólica de su necesidad imaginaria - están los tres términos" (Lacan, 1994:73).

A partir de estos tres términos se podrán introducir todas las variedades. Todo tipo de situaciones ya estructuradas existen entre el niño y la madre. En cuanto la madre se introduce en lo real como potencia, se le abre al niño la posibilidad de un objeto que, como objeto de don, es propiamente intermedio. Por ello, "la cuestión es saber en qué momento y cómo puede ser introducido el niño directamente en la estructura simbólico - imaginario - real, tal como se produce para la madre" (Lacan, 1994:73).

\section{Segundo punto: Sobre el deseo de la madre gira la dinámica de la práctica clínica con niños".}

Para lacan es preciso ubicar el deseo de la "Madre", en la medida en que ella es "Mujer". Según esto se debe considerar a la madre como sujeto correlativo a una falta, no la falta-en-ser, propio del sujeto histérico, sino la falta de un objeto privilegiado que se escribe $\Phi$ (falo). Lacan construye en torno a la falta de objeto una tabla: castración-privación-frustración. Cada uno de estos términos está localizado respecto de lo simbólico - lo real y lo imaginario, como 
un agente distinto, que los coloca en el lugar del objeto faltante. De esta relación Lacan concluye que "lo fundamental para cada sujeto es la relación de la mujer, su madre como su propia falta" (Miller, 1988:237)

J.A. Miller articula en torno a la práctica clínica con niños, la relación de la madre y el niño a través del siguiente matema: $S f$, con $(-\varphi)$

\section{$S n \diamond S f \diamond(-\varphi)$}

En cada análisis de niños es preciso verificar como el sujeto-niño $(S n)$ se articula en relación al sujeto femenino $(S f)$ con su falta fálica, y como se inscribe el niño $(S n)$ en esa relación. Así la elaboración teórica central en el seminario "la relación de objeto" es el término frustración. Y aunque la frustración del niño -ligada a la madre y dependiente de ella- aparezca en primer lugar, "lo más importante es la frustración de la madre como mujer".

Para Lacan la madre es un ser con deseo devorador, insaciable, que nada ni nadie lo puede colmar. En este punto el niño sólo puede obedecer a este término de frustración materna en tanto aparece en la dialéctica intersubjetiva como señuelo. Para satisfacer lo que no puede ser satisfecho, a saber, el deseo de la madre, que en su fundamento es insaciable, el niño por la vía que sea, toma el camino de hacerse él mismo objeto falaz. "Este deseo que no puede ser saciado, es cuestión de engañarlo" (Lacan, 1994:196).

Por esta vía Lacan antepone a la "madre suficientemente buena" de Winnicott (1993), la madre suficientemente deseante. Esa madre insaciable, insatisfecha, a cuyo alrededor se construye toda la ascensión del niño por el camino del narcisismo, es alguien real, "ella está ahí, y como todos los seres insaciables, busca qué devorar querens quem devoret". Lo mismo que el propio niño había encontrado en otro momento para aplastar su insatisfacción simbólica, "vuelve a encontrarlo tal vez frente a él como unas fauces abiertas" (Lacan, 1994:197).

El seminario, "La relación de objeto" (1957), trata de la sexualidad femenina, pero no de la mujer en relación a su goce sino de la mujer en relación al falo, el significante que hace de ella un ser en falta. El niño tapona la falta femenina, pero sólo como un objeto de sustitución. Al igual que la madre y el padre son nombres, el niño es un significante que se puede metaforizar.

$$
\frac{\text { NIÑO }}{(-\varphi)}
$$

La metáfora infantil es en sí misma vacía, no tiene estabilidad para ser autosuficiente. Lacan puntualiza la resolución al enigma del deseo de la madre, en el momento en que el niño descifra que él no es suficiente para colmar el 
agujero materno, y hace depender de esta coyuntura toda la clínica del sujeto: "Cada estructura clínica aparece como un intento de solucionar la deficiencia en cuanto al deseo de la madre" (Miller, 1988:442).

Tanto para Freud como para Lacan el objeto niño no es suficiente para colmar el deseo de la madre, en tanto ésta como mujer siempre está en falta. Ciertamente, el niño tiene relación con la falta, con el falo, pero no más que cualquier otro objeto capaz de hacer desaparecer la falta; es por ello que el significante niño es una construcción metafórica, como tal, objeto de desplazamiento. ¿Quién o qué desplaza al niño como objeto que recubre la falta de objeto en la madre? No siendo el niño como objeto adecuado para colmar esa falta se espera al final la entrada del Nombre-del-Padre.

\section{Tercera tesis: "la metáfora fálica (nombre-del-padre) guarda nexos con la sexualidad femenina"}

Esta metáfora paterna mantiene sus nexos con la sexualidad femenina, en tanto el padre real priva a la madre en el cierre del Edipo, de ese objeto-niño que colma su falta. El rompimiento de este vínculo Lacan lo enuncia así:

"Pero Freud nos revela que es gracias al Nombre-del-Padre como el hombre no permanece atado al servicio sexual de la madre, que la agresión contra el Padre está en el principio de la Ley y que la Ley está al servicio del deseo que ella instituye por la prohibición del incesto" (Lacan, 2009:810).

Este desenlace del Edipo, esta operación de castración que realiza el padre a través de la metáfora del Nombre-del-Padre tiene consecuencias en la clínica a propósito de la teoría fálica: "Pues el inconsciente muestra que el deseo está aferrado al interdicto, que la crisis del Edipo es determinante para la maduración sexual misma" (Lacan, 2009:810). Y, con respecto, a la castración de la mujer sostiene: "(Es el hecho de que la mujer tenga que pasar por la misma dialéctica, cuando nada parece obligarla a ello: necesita perder lo que no tiene, lo que nos pone sobre aviso: permitiéndonos articular que es el falo por defecto el que hace el montaje de la deuda simbólica: cuenta deudora cuando se lo tiene, cuando no se lo tiene, crédito impugnado)" (Lacan, 2009:810).

Lacan construye la metáfora del Nombre-del-Padre a propósito del estudio de los casos clínicos de Freud: "A partir de Juanito (1910), aclara el caso Schreber (1912), en donde aísla el significante fundamental Nombre-delPadre; en tanto que en Juanito aísla la función fálica" (Miller, 1998:116). En la función del significante fóbico opera una suplementación del Nombre-del- 
Padre, y esto porque el niño dispone de este significante primordial. La fobia de Juanito es un pequeño delirio, una minimetáfora delirante construida para suplir el hecho de que el Nombre-del-Padre no está exactamente en su lugar. Es según Lacan, el modo más elegante de dar cuenta de la función del significante comodín, el caballo, que opera en primer plano como muleta de la función del Nombre-del-Padre.

¿Cuáles son los nexos entre esta función del Nombre-del-Padre con el deseo de la madre? Lo esencial de lo que Lacan introduce con Juanito es la madre deseante. Juanito es una exégesis sobre ese estar a solas la madre y el niño, que se supone transcurre en total plenitud. Por ello para Lacan, la pregunta que plantea el análisis de Juanito no es la de la "madre suficientemente buena", sino la de la "madre suficientemente deseante". La madre que cuida y la madre que desea no son las mismas. La lección de Juanito que Lacan aísla es la función de la castración y correlativamente, la identificación virtual del niño con el falo, identificación que es el correlato de la castración materna. El Nombre-del-Padre tiene una función, al respecto, separadora. "De repente la carencia esencial que está en juego es la castración paterna y no la carencia materna" (Miller, 1998:116).

A través del esquema de la metáfora paterna que muestra al Nombre-del-Padre suplantado, metaforizando el deseo de la madre, encontramos debajo de la barra una $x$, el significado del sujeto puede tomar distintas vías: este esquema sirve para la psicosis tanto como para la fobia, para la neurosis e incluso para la perversión.

$\left.\frac{\text { Nombre-del-Padre }}{\text { Deseo de la Madre }} \quad \frac{\text { Deseo de la Madre }}{\text { Significado al sujeto }} \quad \stackrel{\text { Nombre-del-Padre }}{-\varphi}\right]$

Dicho de otra manera, el niño sólo se identifica al tomar del padre la identificación fundamental por la cual asume el deseo de la madre. Así ubica Lacan la metáfora fálica en las tres estructuras clínicas: "Todo el problema de las perversiones consiste en concebir cómo el niño, en su relación con la madre [...] se identifica con el objeto imaginario de ese deseo en cuanto la madre misma lo simboliza en el falo". Esta es la regla para las perversiones" (Lacan, 1981:240).

- En cuanto a la neurosis, Lacan brinda una formula muy general: "aquí la identificación, cualquiera que sea, por la cual el sujeto ha asumido el deseo de la madre". 
- Y en la Psicosis. "Es en el accidente de lo simbólico y de lo que en él se cumple, a saber, la recusación (forclusión) del Nombre-del-Padre en el lugar del Otro, y en el fracaso de la metáfora paterna, donde designamos el efecto que le da a la psicosis su condición esencial, con la estructura que la separa de la neurosis".

- Con la clínica de la marca fálica, de la castración, Lacan presenta una distribución de la clínica asignando su lugar al niño en la medida que se sitúa allí "muy pronto". "Toda la clínica: perversión, neurosis, psicosis se encuentra situada a partir de la consideración del falo, bien distinta a la consideración de los estadios abrahamianos" (Laurent, 1999: 29).

Es verdad que el vacío de la metáfora infantil llama al punto fijo en que puede encarnar al Nombre-del-Padre, pero este tampoco consigue terminar con la falta fálica femenina. "Ese punto clave implica que ha de tomarse en serio el hecho de cómo la pareja de la madre lidia con la falta de ella como mujer" (Miller, 1988:442).

\section{"Cuarta tesis: "hay un final de análisis con niños a partir de la versión del falo y del objeto a"}

Esta doctrina de la "Metáfora fálica" que en sus comienzos Lacan estableció, sirvió a sus alumnos para situar al niño en su posición clásica: el niño como falo. El fin de análisis con era perfectamente deducible de esta doctrina clásica. Según E. Laurent (1999), analizar un niño desde esta posición es asegurarse que tiene una versión del falo, es decir, asegurarse que el niño no es el falo pero que mantiene una relación con él por haberlo sido. Es preciso asegurarse, por una parte, que lo ha sido y, por otra, que no lo sea. Esta versión del falo con la cual termina el niño un análisis es una apuesta por el Padre en el lugar del ideal, el dominio neurótico es hacer creer al niño que él cree en su padre. Para Lacan el fin del análisis a partir de una versión de la teoría fálica no es suficiente, por ello la crítica del falo deviene más y más fuerte a medida que la promoción del objeto $\boldsymbol{a}$ como real se hace insistente.

A partir de los años 1967 y 1969, alrededor de la producción del seminario "El reverso del psicoanálisis", Lacan revalúa la noción de la metáfora paterna, la posición fálica del niño, y la introducción de otras perspectivas que permiten retomar el "muy pronto" que abordó a propósito de una "cuestión preliminar" Esta crítica de la posición fálica del niño y el Edipo freudiano se ordenan en tres textos capitales: en las "Dos notas sobre el niño" (1969), de la carta a Jenny Aubry, y por último en la "Proposición de 1967 sobre psicoanalista de la Escuela". 
En "El reverso del psicoanálisis" (1969), Lacan elabora el matema a la crítica del Edipo articulada en las "Jornadas sobre la infancia alienada": A este respecto comenta, "para comprender cómo situar al niño estamos obligados a tomar en cuenta el tratamiento del goce a una escala que no es la escala familiar y en la que la escala familiar del tratamiento del goce que es la metáfora paterna -el Edipo- está tomada en un orden de subversión mucho mayor". El otro punto de vista importante es la visión del niño generalizado, en este sentido Lacan es revolucionario cuando dice al final del "Discurso de clausura sobre la infancia alienada": Lo que he llegado a creer, fíjese, en ese ocaso de mi vida, le digo, es que "no hay personas mayores" (Laurent, 1999:34).

A partir de esta puntualización no queda ya tan claro que el sitio del niño en el dispositivo analítico sea distinto al del adulto. Si se mira la obra de Lacan, recuerda Laurent, no se encuentra en ningún pasaje el término "adulto", sino la figura de "grandes personnes" (personas mayores); particularizando que la dignidad del psicoanálisis sería la de producir "personas mayores"; en este orden de ideas lo que verdaderamente se podría esperar al final de un análisis sería un sujeto responsable por su goce.

Toda la cuestión del fin de un análisis se podría plantear así: ¿existe un sujeto que sepa lo que ha hecho con su goce? Para el niño no se trata del padre ideal como amo del goce, como amo del deseo; sino de aquel que ha ido hasta el fondo de un deseo y ha encontrado sus restos. A partir de aquí, Lacan va a definir al padre no tanto ya en términos de la relación al falo (Metáfora Fálica), sino en relación al objeto $\boldsymbol{a}$, objeto que causa el deseo de un sujeto. En "El reverso del Psicoanálisis", lo enuncia así: "el objeto $\boldsymbol{a}$ es lo que todos ustedes son, en tanto están ahí cada uno el aborto de lo que fue, para quienes le engendraron, causa del deseo". Es una versión distinta para el niño en un final de análisis, puesto que quiere decir que no se trata del brillo fálico, que no todos están en posición de ser el falo de la madre.

Definir el final de análisis a partir de una versión del falo implica situar al niño a partir de un significante del deseo materno, que es el falo, pero definirlo a partir de una versión del objeto $\boldsymbol{a}$, es situar al niño a partir de un resto. Esta versión del fin de análisis guarda mucho parecido con la versión trágica de "Edipo en Colono", de Sófocles: "el padre deviene entonces aquel que quiere, no prometer el falo, sino enfrentarse con la parte perdida de viviente". Lo que hace que el padre se redefina no es la mortificación fálica sino la vertiente parte perdida. "Edipo en Colono, es el padre que se mortifica en vida, el que entra brutalmente en la muerte, al que el hijo trata de retener cuando se dirige hacia el templo y que desaparece inmediatamente entrando vivo en la muerte" (Laurent, 1999:37). 


\section{Quinta tesis: "el final de análisis con niños implica un tratamien- to del síntoma y su implicación en el fantasma”}

La otra reflexión sobre las vías del final de análisis con niños apunta al tratamiento del síntoma y su relación con el fantasma. A este respecto Serge Cottet (1998), destaca que hoy habría como una "doble doctrina" en los analistas que testimonian sobre la practica con niños: Según el análisis de los niños se conciba como "un medio para alcanzar una meditación simbólica incluso una institución subjetiva del niño en el fantasma, o como operación de desciframiento del goce del síntoma", tesis que implica también -la destitución subjetiva en beneficio del ser para el sexo- (Solano, 1998:13).

\section{Primer punto: "El síntoma tiene que ver con la realidad sexual del sujeto".}

En la "Conferencia de Ginebra sobre el síntoma" (1975), Lacan relee a Freud, para destacar que el síntoma tiene un sentido, el cual está íntimamente ligado a la realidad sexual del sujeto. Comprendido así el síntoma es una forma de circulación del goce sexual por los desfiladeros del significante tal como los traza el inconsciente, es decir la metáfora y la metonimia. ¿Qué relación guarda el síntoma con la realidad sexual del sujeto? Para dar desciframiento a esta pregunta - enigma, Freud, va a buscar la respuesta en la significación de los sueños infantiles, y en la significación de los síntomas a partir de la neurosis traumática. Ante la insuficiencia del concepto de un núcleo traumático universal en el síntoma, y para ampliar una idea a todas la neurosis, Freud lo subordina a otro: el Nachtraglich, la retroactividad en que se define, no es el sentido sino en la significación de lo traumático. A partir de acá se destaca la definición de lo inconsciente como "lo infantil reprimido".

Según Lacan la época de la infancia es "decisiva" porque en ese momento "se cristaliza para el niño lo que hay que llamar por su nombre, es decir los síntomas" luego especifica que el sentido de los síntomas sólo se interpreta correctamente en función de las primeras experiencias del sujeto, "a saber, en la medida que encuentre [. . . ] la realidad sexual". Realidad sexual es un término que Lacan utiliza "por no decir más ni mejor", un signo de que no existe entre "el hombre macho y la hembra ninguna relación instintiva" (Solano, 1998: 11).

\section{Segundo punto: "El síntoma es lo más real que tiene un sujeto".}

El síntoma indica que hay algo que no funciona en el campo de lo real, por ello para el niño hay un encuentro con lo real de lo sexual que se produce de 
forma sintomática. Lacan demuestra tal punto de vista del síntoma a partir de la clínica que se deduce del estudio del caso Juanito: para el niño este encuentro se realiza bajo la incidencia de una primer gozar, el cual se presenta como ajeno a él, le viene del Otro; por ello, este gozar no es en nada auto-erótico, sino de lo más hetero.

Si Lacan puede decir que la sexualidad siempre es traumática, "es porque desde sus primeras experiencias hace valer para el sujeto la antinomia entre el sentido y lo real. Por un lado, el goce incomprendido del niño, que se le presenta a partir de la repentina confrontación con que tiene -un órgano que se mueve-, lo coloca ante lo real de ese goce efectivo que subjetivamente separa al pene de la unidad semántica de su cuerpo, ya que, a ese pene, en tanto sede del goce, el niño, -no consiguió domarlo con sus palabras- ". Por ello, el encuentro con la realidad sexual con lleva un despertar frente a lo insensato del sexo y lo impensable de la muerte. El síntoma muestra la respuesta del sujeto al encuentro traumático de un punto de lo real, puesto que incluye la relación con lo real y el efecto del sentido, y le sirve al sujeto para articular una invención, "algo para llenar el agujero en lo real", como el caballo en la fobia de Juanito (Solano, 1998:11).

De aquí que la neurosis "infantil" sea la solución singular hallada por un sujeto frente al enigma del deseo del Otro. Esta solución supone en cada caso un tipo de síntoma cuya función es hacer existir el inconsciente como saber que se inventa. Para Lacan los sueños y las formaciones del inconsciente muestran por donde se le asegura al sujeto una satisfacción, incluso un goce que puede ser tomado en los carriles de la función fálica, en tanto goce "semiótico" que resulta de la castración operada por el lenguaje.

\section{Tercer punto: "El goce del síntoma está implicado en el fantasma".}

Como la significación no es suficiente para dar cuenta del síntoma, Lacan pone en juego otro elemento: el fantasma. El fantasma interfiere la formación del síntoma y éste es el circuito que enmascara la relación con la pulsión. Por tanto, podemos decir que el síntoma -tal como lo presenta Lacan en "Subversión del sujeto"- está hecho de dos elementos: significación y fantasma. Aparece como una articulación entre efecto significante y la relación del sujeto con el goce. De este modo, lo que empieza a articularse en el grafo del deseo anticipa al síntoma como un nudo, donde están comprometidos el objeto $a$ y un efecto de verdad; ya que, progresivamente, Lacan hará valer, incluido en el fantasma, el objeto a como plus de goce. 
J.A. Miller, en su curso intitulado "Del síntoma al fantasma. Y retorno" (2018), puso muy bien en evidencia la necesidad planteada por Lacan, en la última parte de su enseñanza, de una clínica que se orienta desde el fantasma y no solamente desde el síntoma, implicando, al mismo tiempo, su relación. Veamos ésta articulación: "Decir que el síntoma es un aparato para emparejar al sujeto con el objeto $\boldsymbol{a}$ es casi definirlo como el fantasma" (Miller, 1998:36). El síntoma, en el sentido que articula Lacan, interpone un elemento cultural, el Otro de la cultura, entre esos dos elementos para producir una mediación, para compatibilizarlos, "tratando de reinscribir el síntoma, según su última definición de esas dos coordenadas, propongo poner $\mathbf{s}$ (A) en medio del rombo, y escribir así la definición del síntoma:

$$
\begin{gathered}
\$ \diamond a \\
{[\$<\mathrm{s}(\mathrm{A})>a]}
\end{gathered}
$$

Una mediación entre el sujeto y el goce a través de la significación del Otro". $\mathrm{Al}$ introducir el objeto real en el fantasma, $\$ \diamond a$, articulación del sujeto barrado con el objeto $\boldsymbol{a}$, objeto real causa del deseo, Lacan vuelve a dar al fantasma una causalidad sobre el síntoma. Se despliega así una clínica de la articulación del fantasma con el significante que pasa por la puesta en juego del Otro barrado, portador de una falta fundamental, que es identificada como significante ( $\Phi$ ) falo, mientras que en la clínica del síntoma no ponía en juego más que al Otro no barrado s (A).

\section{Cuarto punto: "Hay un final de análisis con niños ligado a la construc- ción del fantasma"}

Es a propósito del escrito "La proposición al analista de la escuela" (1969), y el seminario titulado "La lógica del fantasma" (1966), de donde surge la controversia sobre el valor de un análisis, en particular la imposibilidad estructural en la clínica con niños de la travesía del fantasma. Lacan plantea allí: "el valor que tiene el psicoanálisis es el de operar sobre el fantasma. El grado de su éxito ha demostrado que ese es el lugar donde se juega la forma que sujeta como neurosis, perversión o psicosis" (Solano, 1993:110). ¿Qué quiere decir operar sobre el fantasma? Lacan advierte a través de estos escritos que el valor de un análisis es el de "operar sobre lo real del goce", desplazar al sujeto respecto a su goce. Por consiguiente, si no hay incidencias analíticas sobre el goce del sujeto no se puede decir que ese análisis tenga un valor.

El impase sexual que suspende al niño hasta el encuentro de la pubertad, nos lleva a interrogarnos por el sustento, es decir, aquello de lo que estaba hecho ese campo del fantasma antes, si puede decirse, de que la connotación 
de verdad afecte, en términos de Lacan "a una proposición que se llamará desde entonces axioma" (Lacan,1991:142). Por ello, si el fantasma está en "función de axioma", la dirección de una cura exige su construcción. Esta, en efecto, no puede reducirse en trabajar solamente las formaciones del inconsciente, incluido el síntoma, puesto que la posición del sujeto en relación con lo que causa su deseo, y mantiene su goce no es modificada por el trabajo sobre las identificaciones significantes. Reducir la intervención solamente por la vertiente del síntoma, es quedarse en el campo de la demanda y de la significación fálica, que representa el niño en el campo del Otro, como objeto del deseo del Otro. Una "construcción” del fantasma implica una posición del niño en la estructura en tanto sujeto, producto de una división, y ello en la medida en que hace uso de la palabra y puede responder por su goce.

En el final de un análisis con el niño, no se puede exceptuar de su condición de ser sexuado, por lo tanto, apto para inscribir su deseo en la lógica de un fantasma, en donde este sujeto-niño está aparejado con el objeto real, objeto $\boldsymbol{a}$ que lo hace gozar; esto supone una posibilidad lógica de acceder a la construcción de un fantasma "fundamental" que opera en la cura, en donde se articula ese valor de goce para el "sujeto sexuado". Ese valor de goce que se advierte en la construcción del fantasma en el final de análisis con el niño, está íntimamente ligado con la figura de la madre, en tanto ella, aparece también como otro ser sexuado. El niño en el fantasma no está sólo, está acompañado por la figura de la madre, sujeto también provisto de un fantasma y como tal susceptible de poder gozar, y que puede encontrar ese objeto real, objeto $\boldsymbol{a}$, causa su goce en la figura del niño. Así entramos a considerar por la vía del fantasma del niño y de la madre los nexos entre final de análisis y sexualidad femenina.

\section{Quinto pinto: "La construcción del fantasma en un final de análisis con niños mantiene sus nexos con la sexualidad femenina"}

A través de Lacan, podemos advertir que las incidencias de la posición del sujeto en la estructura dependen de la forma de posicionarse con respecto al objeto $\boldsymbol{a}$ en el fantasma, y de allí deducimos una forma de goce que habla de la neurosis, la perversión o la psicosis, y en donde el niño se mantiene aferrado a ese valor de goce que representa el deseo materno. Recorramos ahora, lo que hay en la infancia en torno a ese matema $\$$ a que modula las relaciones lógicas entre el sujeto, el objeto y sus nexos con la sexualidad femenina.

Freud nos indica por la vía de la estructura del inconsciente, que el goce del sujeto no depende de su edad, "Tres ensayos de una teoría sexual"; y en "Posición del inconsciente" (1964), Lacan sitúa el advenimiento del sujeto como 
una respuesta a la operación de "enajenación", como el primer movimiento lógico de la causación subjetiva. Por esta operación el sujeto no puede ser causa de sí mismo, al mismo tiempo que haría surgir la dimensión del gran Otro como sede del tesoro de los significantes, esa dimensión del lenguaje que cubre al infantil sujeto.

Esta "enajenación", nos remite entonces inmediatamente a la inadecuación esencial del ser para el sexo, a esa imposible copulación del lenguaje y el cuerpo que Lacan enuncia en el "no hay relación sexual", traduciendo en parte la castración freudiana. Es decir, que el trauma sexual está ya inscrito en la infancia por su dimensión sincrónica en la castración de la madre. Lacan lo enuncia así: "esa falta es inaugural para el niño, que no se despierta a todo lo que se realiza en el acontecimiento psíquico más que al mismo tiempo que descubre con horror que su madre está castrada". Y luego agrega: "formulamos está verdad escribiendo $\mathbf{S}$ (A) como punto de partida en lo que concierne a la lógica del fantasma". S ( $\mathbf{A}$ ) quiere decir que el Otro está marcado. De esto se trata desde el principio en esta castración previa al ser maternal (Lacan, 1993:41).

La segunda operación es la "separación", se reconoce en ella lo que Freud denomino Ichspaltung. Por esta vía el sujeto se realiza en la pérdida en la que ha surgido como inconsciente, por la carencia que se produce en el Otro., "Por las huellas que ha dejado en él la pulsión de muerte" (Lacan; 1981:378). Es decir que, en este segundo movimiento, el sujeto se hace objeto "con lo que tiene a la mano de la parte de sí mismo que le regresa de la enajenación primera". Lo que el sujeto coloca allí, no intenta colmar la faltaen-ser, sino que es recurso contra lo que encuentra de opaco en el deseo del Otro. Lacan en la lógica del fantasma precisa mejor esta segunda operación: "La cuestión de la producción y del estatuto del sujeto como producto, la vemos a nivel de la primera presentificación del Otro que es la madre" (Lacan, 1993:70).

Las consecuencias de este recorrido del viviente que, por el deseo del Otro objetiva su ser de falta, es el fantasma como estructura, como formato de relaciones entre funciones que sostienen su división de sujeto hablante ligándolo así mismo al goce que como objeto $\boldsymbol{a}$, designa la verdad de la estructura.

¿Qué lugar le corresponde al objeto a en este matema (\$ $a)$ ? La respuesta la enuncia Lacan de la siguiente forma: "el objeto $\boldsymbol{a}$, él en tanto pieza separable y profundamente religada al cuerpo es revelo sobre todo de toda una estructura lógica. El objeto a queda como resto de goce por la operación fundamental que ejerce la castración materna, es lo que va a determinar de qué goza un sujeto. En la experiencia de fin de un análisis el objeto a como 
condensador de goce de la madre puede tomar entonces varias formas, según la ubicación del infantil sujeto en la estructura (Lacan; 1993:70).

Este momento del final de análisis implica el punto de arribo para el niño que así realizaría una elección sobre el deseo y no solamente sobre el goce. Acá surge la presencia del falo como ese significante sin igual que permite al niño situarse frente al deseo del Otro. El falo como signo de la castración y razón del deseo, salvaguarda el enigma que constituye el deseo del Otro, articulando en su lugar la pregunta ¿Che voi? A la que se responderá por la vía de la significación fálica.

\section{A modo de conclusión}

Freud deja entrever a través del análisis del caso - "Dora", y el "Hombre de las Ratas" - "la neurosis infantil y sus nexos con la sexualidad Femenina" -, deja entrever el final de análisis como un callejón sin salida que constituye, tanto para el hombre como para la mujer el repudio de la feminidad. La sexuación -versión de la metáfora fálica- implica que el niño sustituya una respuesta a ese callejón sin salida que es el deseo del Otro, en otras palabras, brinde una respuesta a esa pregunta: ¿Qué me quiere el Otro? Tanto para el sujeto masculino como para el sujeto femenino, el encuentro con la feminidad puede constituir un encuentro con lo real. Entendiendo aquí lo real como una versión del falo.

Un complemento a la versión de final de análisis freudiana, se encuentra en un comentario de Lacan a propósito de una "Observación sobre el informe de Daniel Lagache" (1960): "Para llegar a este punto más allá de la reducción de los ideales, es como el objeto a del deseo, como lo que ha sido para el Otro en su erección de vivo, como él wanted o el unwanted de su venida al mundo, como el sujeto está llamado a renacer para saber si quiere lo que desea". Tal es la especie de verdad que con la invención del análisis Freud traía al mundo. "Es este el campo donde el sujeto, con su persona, tiene que pagar sobre todo el rescate de su deseo. Y, en esto es en lo que el psicoanálisis exige una revisión de la ética”. Y, ¿Cuál es la versión del final de análisis que se esperaría para los niños al cierre de una cura? Esta versión se localiza a partir de la función $\Phi$ del significante perdido, a la que el sujeto sacrifica su falo: "para los niños la forma $\Phi$ (a) del deseo masculino, en tanto que para las niñas la forma A $(\varphi)$ del deseo de la mujer", nos indican ese fin de análisis, cuya aporía nos ha legado Freud en la castración. 


\section{Referencias}

Cottet, S. (1998). Freud y el deseo del psicoanalista. Buenos Aires: Editorial Manantial.

Freud, S. (1996). Manuscrito N. Carta 69. En: Obras Completas, Vol. 1. Buenos Aires: Amorrortu Editores. p.p. 296-299.

Freud, S. (1996). Tres ensayos de teoría sexual. En: Obras Completas, Vol. 7. Buenos Aires: Amorrortu Editores. p.p. 109-156.

Freud, S. (1996). "La desfiguración onírica". En: La interpretación de los sueños. Obras Completas. Vol. 5. Buenos Aires: Amorrortu Editores. p.p. 153-180.

Freud, S. (1996). Fragmento de un caso de histeria. En: Obras Completas. Vol.7. Buenos Aires: Amorrortu Editores. p.p. 1-107.

Freud, S. (1996). Analisisis de la fobia de un niño de cinco años. En: Obras Completas. Vol. 10. Buenos Aires: Amorrortu Editores. p.p. 1-118.

Freud, S. (1996). A propósito de un caso de neurosis obsesiva. En: Obras Completas. Vol.10. Buenos Aires: Amorrortu Editores. p.p. 119-172.

Freud, S. (1996). El tabú de la virginidad. En: Obras Completas. Vol.11. Buenos Aires: Amorrortu Editores. p.p. 185-204.

Freud, S. (1996). Sobre un caso de paranoia descrito autobiográficamente (Schreber). En: Obras Completas. Vol. 12. Buenos Aires: Amorrortu Editores. p.p. 1-73.

Freud, S. (1996). Un caso de paranoia que contradice la teoría psicoanalítica. En: Obras Completas. Vol. 14. Buenos Aires: Amorrortu Editores. p.p. 259-272.

Freud, S. (1996). Historia del movimiento psicoanalítico. En: Obras Completas. Vol.14. Buenos Aires: Amorrortu Editores. p.p. 1-64.

Freud, S. (1996). De la historia de una neurosis infantil. En: Obras Completas. Vol. 17. Buenos Aires: Amorrortu Editores. p.p. 1-95.

Freud, S. (1996). Sobre la psicogénesis de un caso de homosexualidad femenina. En: Obras Completas. Vol. 18. Buenos Aires: Amorrortu Editores. p.p. 137-164.

Freud, S. (1996). Algunas consecuencias psíquicas de la diferencia anatómica entre los sexos. En: Obras Completas. Vol. 19. Buenos Aires: Amorrortu Editores. p.p. 259-276.

Freud, S. (1996). El sepultamiento del complejo de Edipo. En: Obras Completas. Vol. 19. Buenos Aires: Amorrortu Editores. p.p. 177-188.

Freud, S. (1996). La organización genital infantil. En: Obras Completas. Vol. 19. Buenos Aires: Amorrortu Editores. p.p. 141-150. 
Freud, S. (1996). La sexualidad femenina. En: Obras Completas. Vol.21. Buenos Aires: Amorrortu Editores. p.p. 223-244.

Lacan, J. (1967). Proposición del 9 de octubre de 1967 sobre el psicoanalista de la escuela. Paris: Inédito.

Lacan, J. (1980). Discurso de Clausura de la jornada de la psicosis en el niño, infancia alienada. Madrid: Editorial Saltés.

Lacan, J. (1981). De una cuestión preliminar a todo tratamiento posible de la psicosis. En: Escritos 2. México: Editorial Siglo XXI.

Lacan, J. (2009). Del Trieb de Freud y el deseo del psicoanalista. En: Escritos 2. México: Editorial Siglo XXI.

Lacan, J. (1981). Observación sobre el informe de Daniel Lagache. En: Escritos 2. Mexico: Editorial Siglo XXI.

Lacan, J. (1981). Posición del inconsciente. En: Escritos 2. México: Editorial Siglo XXI.

Lacan, J. (1988). Conferencia en Ginebra sobre el síntoma. En: Intervenciones y textos 2. Buenos Aires: Editorial Manantial.

Lacan, J. (1988). Dos notas sobre el niño. En: Intervenciones y textos 2. Buenos Aires: Editorial Manantial.

Lacan, J. (1989). Los cuatro conceptos fundamentales del psicoanálisis. Seminario 11. Buenos Aires: Editorial Paidós.

Lacan, J. (1991). La lógica del fantasma. Seminario 14. Buenos Aires: Inédito.

Lacan, J. (1994). La relación de objeto. Seminario 4. Buenos Aires: Editorial Paidós.

Lacan, J. (1999). Las formaciones del inconsciente. Seminario 5. Buenos Aires: Editorial Paidós.

Lacan, J. (2008). El reverso del psicoanálisis. Seminario 17. Buenos Aires: Editorial Paidós.

Laurent, E. (1999). Hay un final de análisis para niños. Buenos Aires: Editorial Diva.

Miller, J. (1998). Recorrido de Lacan. Buenos Aires: Editorial Manantial.

Miller, J. (1988). Elucidación de Lacan. Charlas brasileras. Buenos Aires: EOL-Paidós.

Miller, J. (1988). El síntoma y el cometa. En: El síntoma Charlatán. Barcelona: Editorial Paidós.

Miller, J. (1989). El niño, entre la mujer y la madre. En: Carretel número 1. Buenos Aires.

Miller, J. (2002). De la naturaleza de los semblantes. Buenos Aires: Editorial Paidós. 
Miller, J. (2018). Del síntoma al fantasma. Y retorno. (1982-1983). Buenos Aires: Editorial Paidós.

Recalde, M. (2001). El Edipo un interrogante freudiano. En: Del Edipo a la sexuacion. Buenos Aires: Editorial Paidós.

Silvestre, M. (1987). La neurosis infantil según Freud. En: Mañana el psicoanálisis. Buenos Aires: Editorial Paidós.

Solano, E. (1998). Actualidad de la práctica psicoanalítica. En: Psicoanálisis con niños y púberes. Buenos Aires: Editorial Labrado.

Solano, E. (1998). El síntoma en el niño. En: ¿Cómo cura el psicoanálisis? Bogotá: NEL-Serie Bitácora, vol. 1.

Winnicott, D.W. (1993). Los procesos de maduración y el ambiente facilitador: estudios para una teoría del desarrollo emocional. Buenos Aires: Editorial Paidós. 